\title{
Re: Prospective, Randomized, Multinational Study of Prostatic Urethral Lift Versus Transurethral Resection of the Prostate: 12-month Results from the BPH6 Study
}

\author{
Jens Sønksen1, Neil J. Barber2, Mark J. Speakman3, Richard Berges4, Ulrich Wetterauer5, \\ Damien Greene6, Karl-Dietrich Sievert7, Christopher R. Chapple8, Francesco Montorsi9, \\ Jacob M. Patterson8, Lasse Fahrenkrug1, Martin Schoenthaler5, Christian Gratzke10
}

1 Herlev Hospital, Department of Urology, Herlev, Denmark

2Frimley Park Hospital NHS Foundation Trust, Surrey, UK

3 Musgrove Park Hospital, Department of Urology, Taunton and Somerset NHS Trust, Taunton, UK

4PAN Klinik Köln, Department of Urology, Köln, Germany

5 University Hospital Freiburg, Department of Urology, Freiburg, Germany

6 City Hospitals Sunderland, Sunderland, UK

7 University Clinic of Lübeck, Lübeck, Germany

8Sheffield Teaching Hospitals NHS Foundation Trust, Sheffield, UK

9Instituto San Raffaele, Urology Division Milan, Italy

10Ludwig-Maximilians University, Department of Urology, Munich, Germany

Eur Urol 2015;68:643-652.

\section{EDITORIAL COMMENT}

Prostatic urethral lift (PUL) has been recently introduced as a minimally invasive procedure for relieving benign prostatic hyperplasia (BPH) associated lower urinary tract symptoms (LUTS) while preserving sexual functions. BPH6 study is the first prospective, randomized study comparing PUL with transurethral resection of the prostate (TURP) which still represents the standard surgical procedure for BPH. The authors proposed a novel evaluation method for comparing treatment outcomes which they named BPH6 composed of 6 items including symptom relief, high-quality recovery, erectile functions, ejaculatory functions, continence and high-grade complications. The study included 80 patients (45 PUL, 35 TURP) with a follow-up period of 12 months. With similar baseline characteristics, the authors reported significantly higher rate of achieving the BPH6 recovery endpoint by 1 month in the PUL group compared to TURP group. Discharge from hospital and returning to preoperative activity level were in favor of PUL group. Despite significant improvement concerning the International Prostate Symptom Score (IPSS), IPSS-Quality of Life (IPSS-QoL), BPH-II and the peak urinary flow rate (Qmax) in both treatment arms compared to baseline; TURP was much better than PUL. Erectile functions evaluated by the Sexual Health Inventory for Men (SHIM) were preserved in both treatment arms. The authors reported improved ejaculatory functions (MSHQ-EjD) for the PUL group, whereas there was a significant decline in TURP group. Continence preservation was comparable between the groups. None of the patients in the PUL group required surgical intervention for any adverse event, however, 2 patients in the TURP group required endoscopic intervention. PUL seems to have no significant effect on sexual functions while improving LUTS in short-term follow-up, however, further trials with long-term follow-up are required. 